\title{
A three-gene novel predictor for improving the prognosis of cervical cancer
}

\author{
TING-TING DING ${ }^{1}$, HU MA ${ }^{1}$ and JI-HONG FENG ${ }^{2}$ \\ ${ }^{1}$ Department of Oncology, Tumor Hospital, Affiliated Hospital of Zunyi Medical University, \\ Zunyi, Guizhou 563000; ${ }^{2}$ Department of Oncology, Taizhou Municipal Hospital, \\ The Affiliated Hospital of Medical College of Taizhou University, Taizhou, Zhejiang 318000, P.R. China
}

Received January 20, 2019; Accepted August 16, 2019

DOI: $10.3892 / \mathrm{ol} .2019 .10815$

\begin{abstract}
Cervical cancer is the most common gynecological malignancy, the third most common malignant tumor in women worldwide, and the most common malignant tumor among Chinese women. However, despite continuous improvement in medical treatment, the number of cervical cancer cases in China is on the increase annually, consistent with the general trend in global cervical cancer incidence. Therefore, it is particularly important to study the pathogenesis of cervical cancer at the genetic level in China. The aim of the present study was to use the TCGA database to identify potential genetic signatures that could predict the prognosis of patients with cervical cancer and provide evidence supporting clinical genetic intervention in cervical cancer. Primarily, an effective three-gene signature was found that predicts prognosis in patients with cervical cancer. This model can provide prima facie evidence for future assessment of patient risk and prognosis, but further testing is required to improve its accuracy. Our results also suggested that centromere protein M, methionine sulfoxide reductase B3 and Zic family member 2 could be promising biomarkers for the prognosis of cervical cancer.
\end{abstract}

\section{Introduction}

Cervical cancer is one of the most common cancers among women. According to statistics reported in 2018, $\sim 528,000$ patients worldwide are diagnosed with cervical cancer each year (1). Global tumor statistics show that cervical cancer ranks fourth in morbidity and mortality among female malignancies worldwide; approximately $85 \%$ of cervical cancer deaths worldwide occur in less-developed or developing countries, and cervical cancer mortality in low- and middle-income

Correspondence to: Professor Hu Ma, Department of Oncology, Tumor Hospital, Affiliated Hospital of Zunyi Medical University, 201 Dalian Road, Zunyi, Guizhou 563000, P.R. China

E-mail:mahuab@163.com

Key words: gene signature, cervical cancer, prognostic signature, risk score countries is 18 times higher than in high-income countries (2). The incidence of cervical cancer in China is ranked second worldwide only to Chile in 2015, and its prevalence in younger women is notable; 93 women die of cervical cancer every day (3). Efforts to prevent cervical cancer, in areas of China with low economy, and to improve morbidity and mortality in patients with cervical cancer requires improvement in global cancer treatment regimens. Despite the increasing popularity of and diagnostic techniques for early screening of cervical cancer, the number of new cases in China is 13.15 million per year, accounting for $28.8 \%$ of new cases worldwide in 2019, and shows an incidence trend in younger age groups (35-39 years) (4). The occurrence and development of cervical cancer is affected by various regulatory factors. A study in China and worldwide revealed that persistent infection with high-risk human papillomavirus (HPV) was closely related to the pathogenesis of cervical cancer (5). Mutations in some genes, such as p53, p16, and Nm23, may also cause malignant transformation of cells (6). Biomarkers are biochemical indicators that can label changes in systems, organs, tissues, and cells (6). To the best of our knowledge, few studies on cervical cancer genes have been conducted. One of the main limiting factors of better diagnosis and treatment of cervical cancer is the lack of knowledge and determination of effective molecular markers (7). Therefore, the identification of specific molecules has become a research hotspot. The present study explores cervical cancer-related genes based on the original data in public databases and provides novel ideas for early cervical cancer research.

In recent years, as gene chips and sequencing technologies continue to evolve, high-throughput data have burgeoned. The Cancer Genome Atlas (TCGA) and the human tumor-associated Gene Expression Omnibus databases are two large public databases that provide high-throughput data for a variety of diseases, such as lung and breast cancer, for analysis (8). The introduction of TCGA database in 2005 through a new genomic analysis technology deepened researchers' comprehensive understanding of cancer genetics and provides an open dataset to assist with generating new cancer treatments, diagnostic methods and prevention strategies (9). As a publicly funded project, TCGA database aims to distinguish approximate genomic variations in cancer to create a comprehensive 'cancer genomic profile' map. Currently, TCGA database 
researchers have studied a total of 14,551 cumulative cases of $>30$ types of cancer in humans (such as lung cancer, cervical cancer and colorectal cancer) through large-scale genome sequencing and comprehensive multidimensional analysis. Based on TCGA database, the present study used the $\mathrm{R}$ language to mine 654 differentially expressed genes (DEGs) in cervical cancer tissues and combined this approach with bioinformatics software and literature mining methods to define the functions and pathways enriched in the DEGs. Combining 304 cervical cancer clinical samples from the TCGA database and analysing the prognosis of the patients, important information for studying the molecular mechanism of genes in the occurrence and development of cervical cancer was assembled and provided novel ideas and targets for the future treatment of cervical cancer.

\section{Materials and methods}

Screening of DEGs. TCGA database (https://cancergenome. nih.gov/) was used to download raw sequencing data and clinical information. The standardized datasets were obtained by annotation and integration. Perl (http://www.perl.org/) scripting tools were used to perform integration processing of datasets. Analysis of DEGs between cervical cancer and normal tissues was conducted with the $\mathrm{R}$ language package (version 3.5.1; Shengxin Self-study Network); differential expression was defined as a $\log _{2}$ fold change (FC) of $>4$ and $P$ adj=0.001). The data for clinical survival times and the expression of DEGs were merged using Perl scripting tools, and the prognostic value of each DEG was subsequently evaluated using the log-rank method.

Gene ontology (GO) and Kyoto encyclopedia of genes and genomes (KEGG) pathway enrichment analysis. In the present study, a total of 654 DEGs were screened from cervical cancer samples. The 654 DEGs were subjected to KEGG analysis (https://www.kegg.jp/) and GO enrichment analysis (http:// geneontology.org). As a result, 195 genes were enriched into the KEGG signaling pathway, and 246 genes were enriched in GO and subsequently visualized to obtain KEGG map and GO map, respectively. KEGG is a database resource for understanding the advanced functions and activities of biological systems, especially from large-scale molecular datasets generated by genome sequencing and other high-throughput experimental techniques. The KEGG database was used to perform pathway enrichment analysis on the identified DEGs. The RSQLite program in the R package was used to assist the transformation of DEGs into Entrez IDs. Then, the enrichment data were visualized using Cytoscape software (https:// cytoscape.org/). GO was used to describe gene functions and relationships between these concepts; GO annotations are statements that use the concept of gene ontology to describe the function of a particular gene $(10,11)$. DEGs were uploaded to the Database for Annotation, Visualization and Integrated Discovery website (https://david.ncifcrf.gov/) to obtain the gene enrichment results, and the results were then visualized with the R language package as the $\mathrm{GO}$ analysis results.

Three-gene signature and risk score assessment. DEGs were screened using single-factor Cox regression analysis using the
Table I. Number of cases and proportion of each clinical feature of patients with cervical cancer.

\begin{tabular}{|c|c|}
\hline Variable & Case, n (\%) \\
\hline \multicolumn{2}{|l|}{ Age } \\
\hline$\leq 46$ & $154(51.2)$ \\
\hline$>46$ & $147(48.8)$ \\
\hline \multicolumn{2}{|l|}{ Grade } \\
\hline $\mathrm{G} 1+\mathrm{G} 2$ & $152(50.5)$ \\
\hline $\mathrm{G} 3+\mathrm{G} 4$ & 117 (38.9) \\
\hline GX & $24(7.9)$ \\
\hline NA & $8(2.7)$ \\
\hline \multicolumn{2}{|l|}{ T stage } \\
\hline Tis & $1(0.3)$ \\
\hline $\mathrm{T} 1+\mathrm{T} 2$ & $209(69.4)$ \\
\hline $\mathrm{T} 3+\mathrm{T} 4$ & $30(10.0)$ \\
\hline $\mathrm{TX}$ & $17(5.6)$ \\
\hline NA & $44(14.6)$ \\
\hline \multicolumn{2}{|c|}{ Lymph node status } \\
\hline No & $132(43.9)$ \\
\hline N1 & $59(19.6)$ \\
\hline NX & $66(21.9)$ \\
\hline NA & $44(14.6)$ \\
\hline \multicolumn{2}{|c|}{ Metastasis } \\
\hline M0 & 115 (38.2) \\
\hline M1 & $10(3.3)$ \\
\hline MX & $128(42.5)$ \\
\hline NA & $48(15.9)$ \\
\hline
\end{tabular}

NA, not available; X, cannot be determined/data uncertain; Tis, carcinoma in situ.

Survival package to screen out DEGs associated the overall survival (OS) of patients. Multivariate Cox regression analysis was used to establish a multigene prognosis prediction model to derive three-gene signatures and calculate risk scores as follows: Risk score $=\Sigma \beta_{\text {genei }} \times \operatorname{Exp}_{\text {genei }}(\mathrm{i}=1-\mathrm{N})=\beta_{\text {genel }} \times \operatorname{Exp}_{\text {genel }}$ $+\beta_{\text {gene2 }} \times \operatorname{Exp}_{\text {gene } 2}+\ldots+\beta_{\text {geneN }} \times \operatorname{Exp}_{\text {geneN }}(12)$; where $\beta$ represents the regression coefficient, Exp represents the risk ratio, i represents the running formula, and $\mathrm{N}$ represents the total number of samples. Patients were divided into a high-risk group and a low-risk group according to the median score. The effect of each gene on patient survival was then assessed using the Kaplan-Meier method. The receiver operating characteristic (ROC) curve was plotted, and the survival ROC package in the $\mathrm{R}$ language was used to estimate the area under the curve (AUC).

Statistical analysis. All the data in the present study were analyzed using R software (version 3.5.1) and Perl scripting tools. Continuous variables are presented as mean $\pm \mathrm{SD}$, while categorical variables are presented as frequencies and percentages. The relationship between gene expression and clinical features was assessed using $\chi^{2}$ and Student's t-test. 


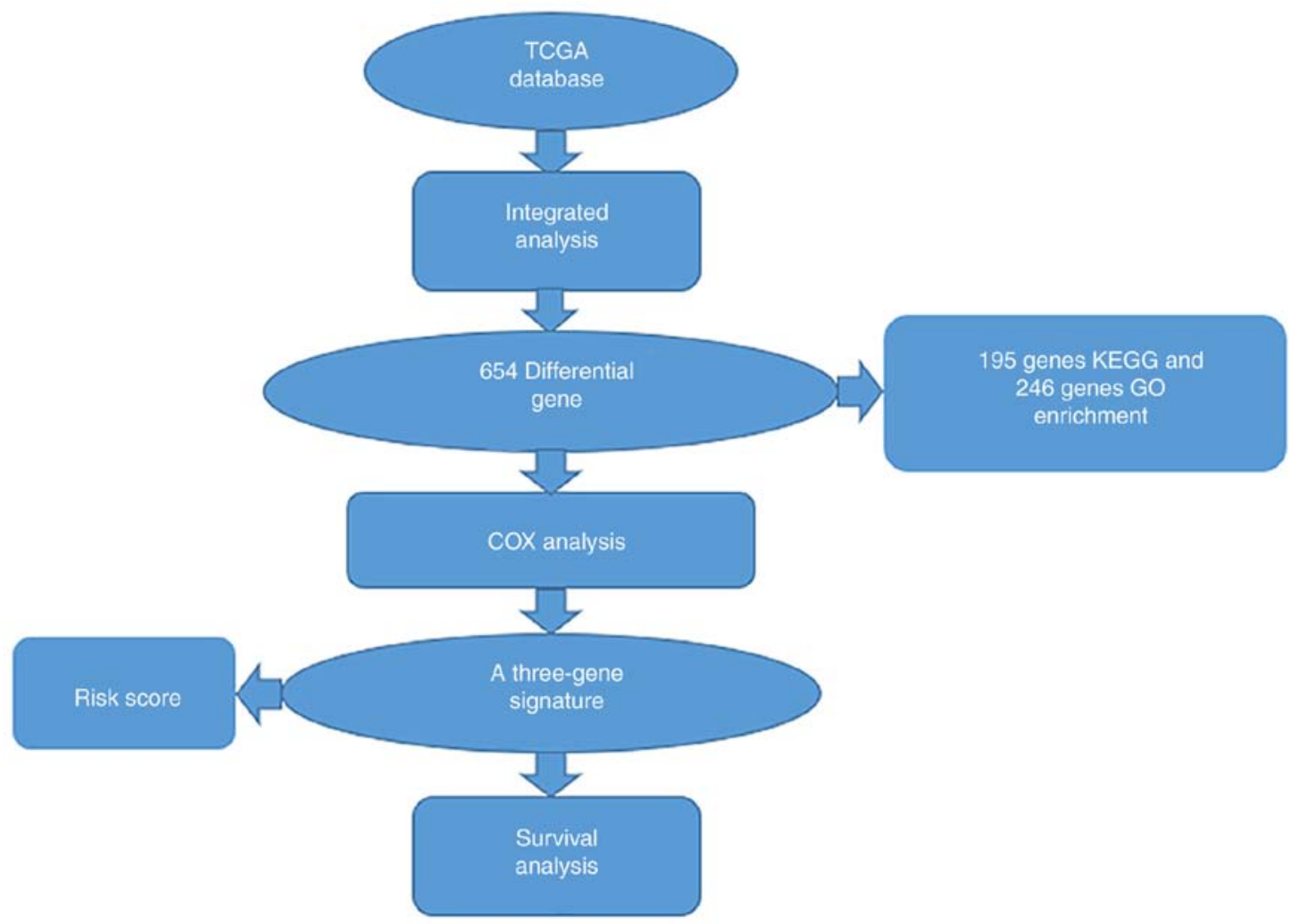

Figure 1. Screening of the three genes and analysis of related pathways affecting prognosis. Genomic mRNA-seq expression data and clinical cervical cancer materials were downloaded from the TCGA database website. Three-gene characteristics were obtained by univariate and multivariate Cox regression analysis. TCGA, The Cancer Genome Atlas; KEGG, Kyoto Encyclopedia of Genes and Genomes; GO; Gene Ontology.

Each risk gene and prognosis gene marker were analyzed using Kaplan-Meier survival analysis and univariate/multivariate Cox proportional hazards regression. $\mathrm{P}<0.05$ (two-tailed) were considered to indicate a statistically significant difference.

\section{Results}

Workflow presentation. A series of combinatorial analysis were used to identify DEGs in cervical cancer and to screen for three-gene signatures associated with cervical cancer prognosis through a variety of methods (Fig. 1).

Screening of DEGs in cervical cancer samples. Data for 304 patients with cervical cancer and for 309 cervical cancer tissue samples were downloaded from TCGA database, however only 301 cases were matched between the clinical data and tissue sample data. A total of three of the tissue samples did not have associated clinical data, and 3 patients with clinical data did not have associated tissue samples; these samples were excluded. The 309 cervical cancer tissue samples included 306 cervical cancer tumor tissues and 3 normal tissues from healthy controls. The detailed characteristics of the clinical data were stratified according to age, grade, T stage, lymph node status and metastasis, as shown in Table I. The results of the differential gene expression in cervical cancer are displayed as a volcano plot and show whether the $\mathrm{P}$ - and $\log _{2} \mathrm{FCl}$ values satisfied the logic of different tests (Fig. 2). A total of 301 cervical cancer samples downloaded from TCGA database were analyzed for differential gene

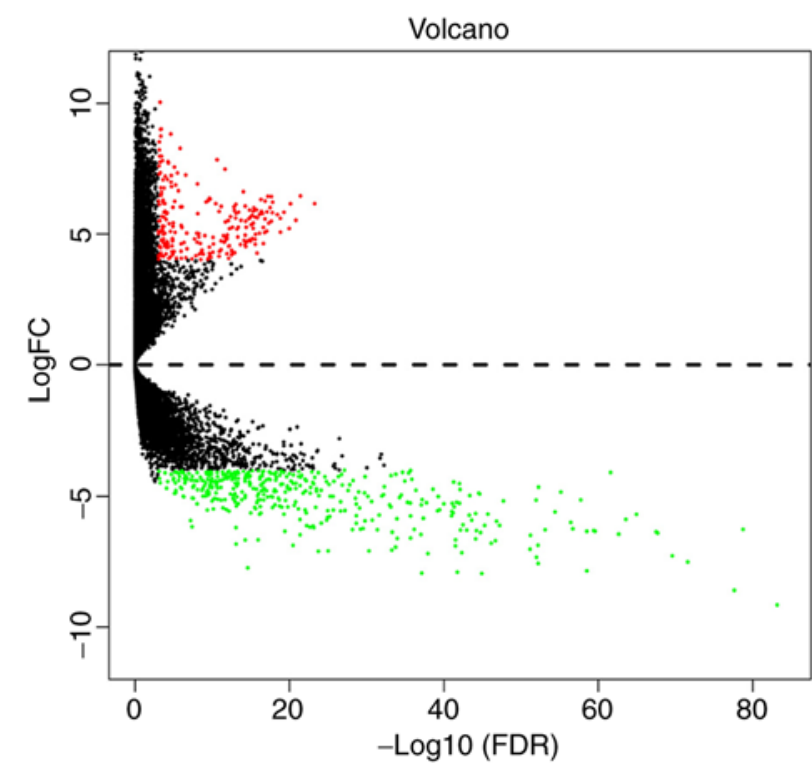

Figure 2. Volcano plot of DEGs. A total of 654 DEGs were identified in cervical cancer samples downloaded from The Cancer Genome Atlas database. The red dots indicate upregulated genes, the green dots indicate downregulated genes, and the black dots indicate the absence of statistically significant genes. DEGs, differentially expressed genes; FC, fold change; FDR, false discovery rate.

expression, resulting in the identification of 654 DEGs, among which 239 were upregulated and 415 were downregulated (fold change $>4, \mathrm{P}$ adj $=0.001$ ). 
Table II. Significantly enriched KEGG pathway of differential expressed genes.

\begin{tabular}{|c|c|c|c|c|c|c|}
\hline ID & Description & GeneRatio & BgRatio & P-value & Adjust P-value & Count \\
\hline hsa04110 & Cell cycle & $22 / 195$ & $124 / 7469$ & $6.20 \times 10^{-13}$ & $1.32 \times 10^{-10}$ & 22 \\
\hline hsa04270 & Vascular smooth muscle contraction & $16 / 195$ & $121 / 7469$ & $8.12 \times 10^{-8}$ & $8.65 \times 10^{-6}$ & 16 \\
\hline hsa04115 & p53 signaling pathway & $10 / 195$ & $72 / 7469$ & $1.53 \times 10^{-5}$ & $8.81 \times 10^{-5}$ & 10 \\
\hline hsa04614 & Renin-angiotensin system & $6 / 195$ & $23 / 7469$ & $2.04 \times 10^{-5}$ & $8.81 \times 10^{-5}$ & 6 \\
\hline hsa04114 & Oocyte meiosis & $13 / 195$ & $125 / 7469$ & $2.07 \times 10^{-5}$ & $8.81 \times 10^{-5}$ & 13 \\
\hline hsa04022 & cGMP-PKG signaling pathway & $14 / 195$ & $163 / 7469$ & $8.50 \times 10^{-5}$ & $3.02 \times 10^{-3}$ & 14 \\
\hline hsa04080 & Neuroactive ligand-receptor interaction & $19 / 195$ & $277 / 7469$ & $10.5 \times 10^{-5}$ & $3.19 \times 10^{-3}$ & 19 \\
\hline hsa04914 & Progesterone-mediated oocyte maturation & $10 / 195$ & $99 / 7469$ & $24.2 \times 10^{-5}$ & $6.43 \times 10^{-3}$ & 10 \\
\hline hsa04020 & Calcium signaling pathway & $14 / 195$ & $183 / 7469$ & $2.9 \times 10^{-4}$ & $6.86 .4 \times 10^{-3}$ & 14 \\
\hline hsa05412 & $\begin{array}{l}\text { Arrhythmogenic right ventricular } \\
\text { cardiomyopathy }\end{array}$ & $8 / 195$ & $72 / 7469$ & $5.35 \times 10^{-4}$ & $1.14 \times 10^{-2}$ & 8 \\
\hline hsa03440 & Homologous recombination & $6 / 195$ & $41 / 7469$ & $6.15 \times 10^{-4}$ & $1.19 \times 10^{-2}$ & 6 \\
\hline hsa03460 & Fanconi anemia pathway & $6 / 195$ & $54 / 7469$ & $2.67 \times 10^{-3}$ & $4.74 \times 10^{-5}$ & 6 \\
\hline
\end{tabular}

GeneRatio, the ratio of the number of genes associated with the ID to the total number of genes in the differential gene; BgRatio, number of genes of the corresponding species/the number of genes in the KEGG pathway database containing the corresponding species.

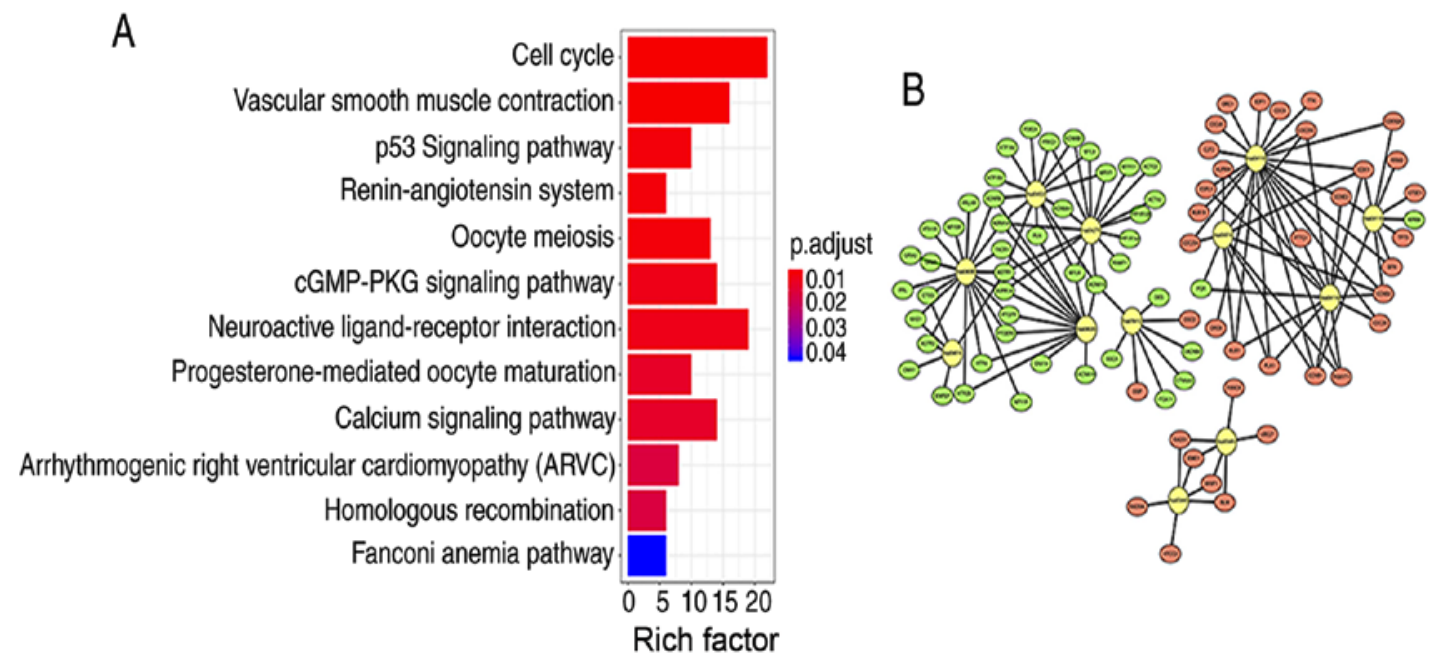

Figure 3. KEGG enrichment map of differentially expressed genes. (A) The bar graph shows the top 12 KEGG pathways. Smaller P-values and deeper red color indicates higher enrichment $(\mathrm{P}<0.05 ; \mathrm{n}=195)$. (B) Cytoscape software was used to display an interactive network map of the top $12 \mathrm{KEGG}$ functional pathways. KEGG, Kyoto Encyclopedia of Genes and Genomes.

KEGG enrichment analysis and signaling pathway visualization. Signaling pathway enrichment analysis of the DEGs was conducted using the KEGG database. The 654 differentially expressed genes in cervical cancer samples were analyzed by KEGG enrichment, and the results of 195 enriched genes were statistically significant $(\mathrm{P}<0.05)$. The KEGG analysis results showed that the 195 DEGs were significantly associated with 12 signaling pathways $(\mathrm{P}<0.05$; Table II). The top five signaling pathways with the strongest enrichment in the KEGG analysis were as follows: hsa04110 ('Cell cycle'); hsa04270 ('vascular smooth muscle contraction'); hsa04115 ('p53 signaling pathway'); hsa04614 ('renin-angiotensin system') and hsa04114 ('oocyte meiosis'). Next, R package components were used to construct KEGG signaling pathway diagrams (Fig. 3A). The larger the value of Rich factor value, the greater the enrichment. Rich factor=number of differentially expressed genes located under the pathway term/number of all annotated genes located under the pathway term. The enrichment analysis data were visualized using Cytoscape software (Fig. 3B).

Functional analysis of DEGs via GO enrichment analysis. $\mathrm{GO}$ analysis was performed on all 654 differentially expressed genes, of which 246 genes were enrichment and statistically significant $(\mathrm{P}<0.05)$. The $246 \mathrm{DEGs}$ were also analyzed for GO term enrichment using the Gene Ontology Enrichment Analysis Software Toolkit. The following GO biological process terms were mainly enriched in the DEGs: Regulation of mitotic nuclear division, regulation of cell division and regulation of sister chromatid cohesion (Table III). The main 
Table III. Enrichment analysis of differentially expressed genes in the Gene Onotology enrichment analysis.

\begin{tabular}{llcl}
\hline ID & \multicolumn{1}{c}{ Biological function } & Count & P-value \\
\hline GO:0007067 & Mitotic nuclear division & 36 & $1.30 \times 10^{-15}$ \\
GO:0051301 & Cell division & 39 & $4.01 \times 10^{-13}$ \\
GO:0007062 & Sister chromatid cohesion & 21 & $4.58 \times 10^{-12}$ \\
GO:0000082 & $G_{1} /$ S transition of mitotic cell cycle & 16 & $1.12 \times 10^{-7}$ \\
GO:0007059 & Chromosome segregation & 13 & $2.74 \times 10^{-7}$ \\
GO:0008283 & Cell proliferation & 30 & $3.10 \times 10^{-7}$ \\
GO:0000777 & Condensed chromosome kinetochore & 14 & $5.72 \times 10^{-7}$ \\
GO:0030018 & Z disc & 16 & $6.73 \times 10^{-7}$ \\
GO:0006936 & Muscle contraction & 15 & $1.27 \times 10^{-6}$ \\
GO:0005876 & Spindle microtubule & 10 & $2.17 \times 10^{-6}$ \\
GO:0000775 & Chromosome, centromeric region & 11 & $2.60 \times 10^{-6}$ \\
GO:0000070 & Mitotic sister chromatid segregation & 8 & $3.42 \times 10^{-6}$ \\
GO:0005578 & Proteinaceous extracellular matrix & 23 & $3.98 \times 10^{-6}$ \\
GO:0005819 & Spindle & 15 & $4.90 \times 10^{-6}$ \\
GO:0000086 & $G_{2} /$ M transition of mitotic cell cycle & 16 & $5.19 \times 10^{-6}$ \\
\hline
\end{tabular}

Table IV. Multivariate Cox regression analysis in a three-gene signature

\begin{tabular}{lccccc}
\hline Gene & Coef & Exp (coef) & SE (coef) & $\mathrm{z}$ & P-value \\
\hline MSRB3 & 0.18134 & 1.19882 & 0.08608 & 2.107 & $3.51 \times 10^{-2}$ \\
CENPM & 0.45186 & 0.63644 & 0.16121 & -2.803 & $5.06 \times 10^{-3}$ \\
ZIC2 & 0.11147 & 0.89452 & 0.05007 & -2.226 & $2.60 \times 10^{-2}$ \\
\hline
\end{tabular}

Likelihood ratio test, 48.04; $\mathrm{P}=3.484 \times 10^{-9} ; \mathrm{n}=304$; number of events, 71. Coef, regression coefficient; Exp (coef), risk ratio; SE (coef), standard error, $z$, ratio of each regression coefficient to its standard error $[\mathrm{z}=\mathrm{Coef} / \mathrm{SE}($ coef $)]$.

biological functions of the DEGs studied that are enriched, the number of enriched genes and the degree of enrichment of different GO terms arepresented in Fig. 4A. The DEGs in the top $5 \mathrm{GO}$ terms with the highest enrichment level (the top 5 with the lowest P-value) demonstrated the highest degree of enrichment are shown; the genes marked in red were upregulated, while those marked in blue were downregulated (Fig. 4B).

Detection of three-gene signatures and risk scores. Three specific genes were screened using univariate survival analysis and Cox regression analysis, and the significant association of three genes as independent predictors of OS were validated $(\mathrm{P}<0.05)$. This three-gene signature comprised methionine sulfoxide reductase B3 (MSRB3), centromere protein M (CENPM) and Zic family member 2 (ZIC2). The risk score for each patient was calculated based on the sum of the weighted expressions of the three genes (likelihood ratio test, 48.04; $\mathrm{P}=3.484 \times 10^{-9} ; \mathrm{n}=304$; Table IV). The result of the analysis revealed that the higher the risk score, the worse the clinical prognosis. To identify possible genes associated with OS in patients with cervical cancer, Kaplan-Meier curves and the log-rank test were used to evaluate the association between gene expression and patient survival. As shown in Fig. 5, the expression of CENPM and ZIC2 was positively associated with the survival rate of patients with cervical cancer, while the expression of MSRB3 was negatively associated with the survival rate of patients with cervical cancer.

Verification of the regression model and risk degree. Each patient from the dataset was assigned to the high-risk group or the low-risk group based on their risk score. In the present study, the median risk score was calculated to be 1.33 for the 304 patients based on the risk score formula, 152 patients with the score $<1.33$ were included in the low-risk group, and 152 patients with the score $\geq 1.33$ were included in the highrisk group. The analysis results indicated that the risk score was significantly associated with $\mathrm{OS}(\mathrm{P}<0.05)$ as a prognostic factor in univariate regression analysis. The results also showed that the risk score was an independent prognostic factor for OS in multivariate Cox regression analysis $(\mathrm{P}<0.05$; Fig. 6A). In addition, the ROC curve and AUC (0.782) analysis results showed high sensitivity and specificity $(\mathrm{P}<0.05$; Fig. $6 \mathrm{~B})$.

\section{Discussion}

Cervical cancer was reported as the third most common gynecological malignancy worldwide in 2012 (13). In China, the incidence of cervical cancer ranks first among malignant tumors in the female reproductive system (14), and $>99 \%$ of patients with cervical cancer are infected with HPV $(11,15,16)$. In 2011, the American Cancer Society, the American Society for Colposcopy and Cervical Pathology, and the American Society for Clinical Pathology updated their joint guidelines for cervical cancer screening, as well as the US Preventive Services Task Force (17-19). It can be seen that cervical cancer screening for the early detection and prevention of cervical cancer is crucial to reduce the incidence and mortality of cervical cancer in China. In addition, understanding the 

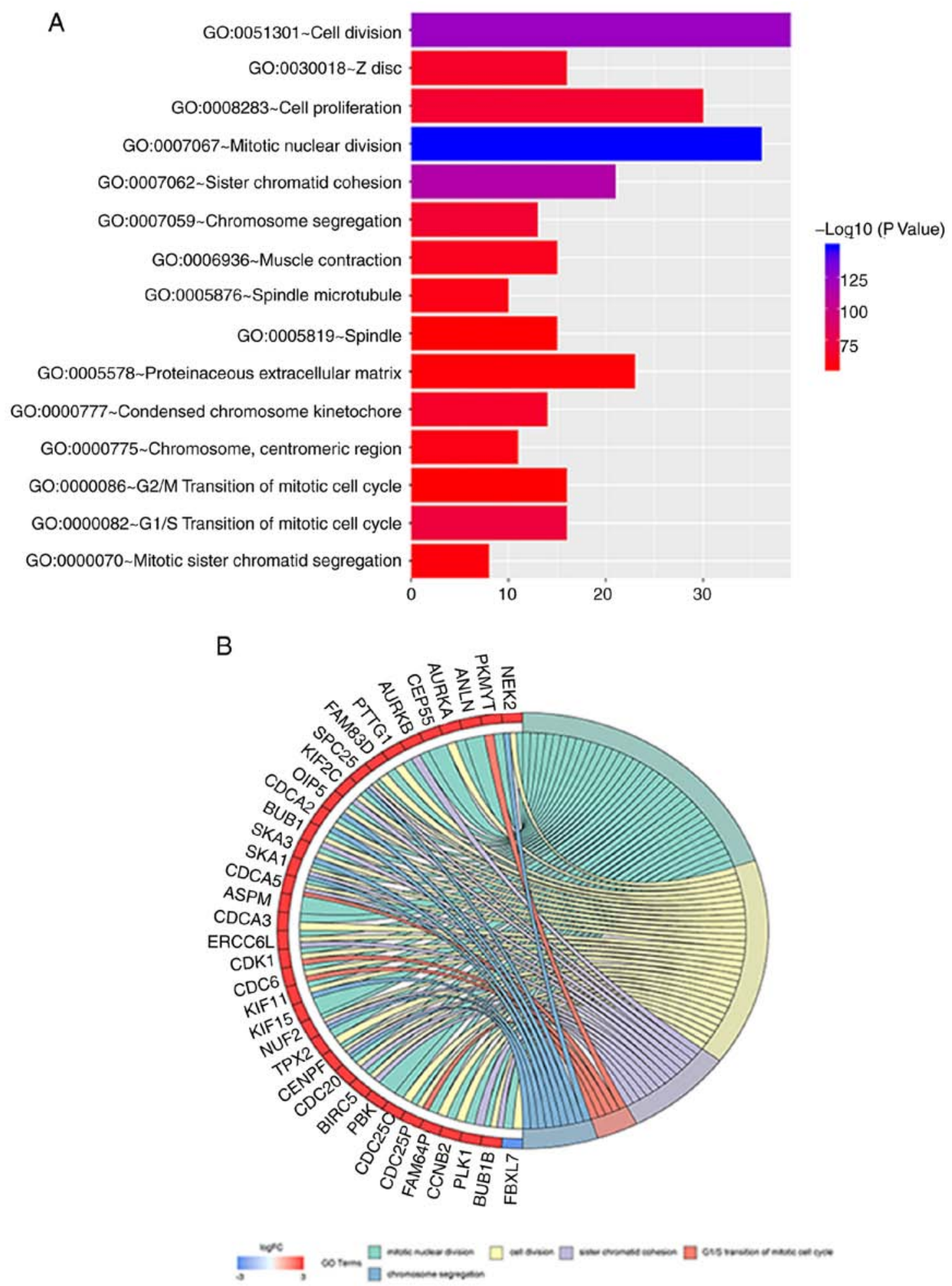

Figure 4. Target gene prediction and functional analysis. (A) The top fifteen biological process GO terms enriched in the genes of interest. (B) Upregulated and downregulated genes in the top 5 most enriched GO terms. GO, Gene Ontology; FC, fold change.

pathological mechanisms and finding potential survivalrelated genes involved in cervical cancer development is a direction requiring diligent study. With the latest developments in biotechnology, an increasing number of technologies, such as gene sequencing, transcriptome analysis, DNA methylation and epigenetic analysis, are being used to study tumor genomes (20). In the present study, TCGA database was used to analyze normal tissues from healthy controls and tumor tissues of patients with cervical cancer to determine the DEGs in tumor tissues. Then, the three-gene signature and risk scores were verified by univariate and multivariate Cox regression analysis, and the risk scores were found to be significantly associated with OS. It can be concluded that our three-gene signature can predict the pathogenesis of cervical cancer at the genetic level. Therefore, our research results not only provide an experimental background for future experiments on 

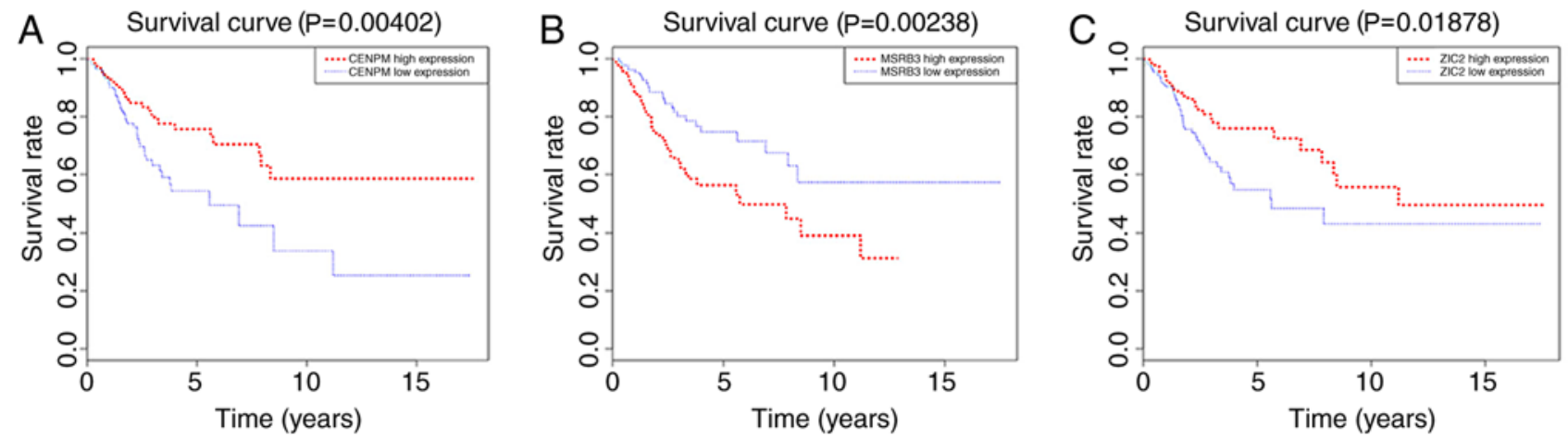

Figure 5. Association between the three genes and the survival of patients with cervical cancer was assessed using Kaplan-Meier curves and the log-rank test. The patients were stratified into high- and low-risk groups according to the median risk score of (A) CENPM (P<0.01), (B) MSRB3 (P<0.01) and (C) ZIC2 $(\mathrm{P}<0.05)$. CENPM, centromere protein M; MSRB3, methionine sulfoxide reductase B3; ZIC2, Zic family member 2.
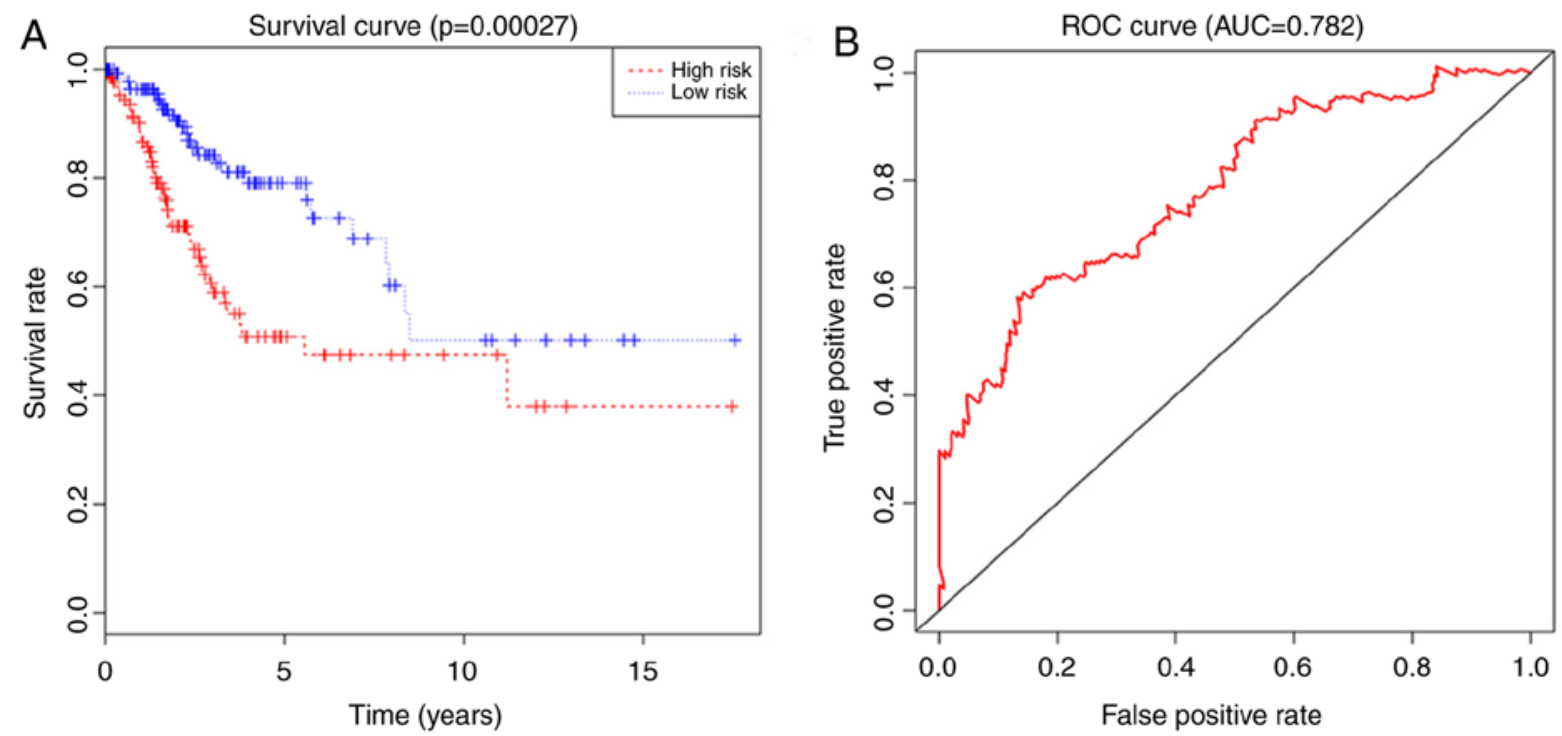

Figure 6. Gene signature risk score analysis results. (A) The risk score was significantly associated with OS ( $<<0.001)$. The risk score was an independent prognostic factor for OS in multivariate Cox regression analysis. (B) The ROC curve was used to assess the diagnostic ability of the gene signature in terms of specificity and sensitivity. The AUC was 0.782 , indicating that the three gene markers are highly sensitive and specific for the cervical cancer classification of normal controls. OS, overall survival; ROC, receiver operator characteristic; AUC, area under the curve.

cervical cancer but also provide strong evidence for prognostic evaluation of cervical cancer in a clinical setting.

The three genes (MSRB3, CENPM and ZIC2) were found to function both as risk factors and protective factors. The molecular functions of these three genes in other tumors, will provide a reference for further studies on these genes in cervical cancer. MSRB 3 is a member of the MSR family of proteins (21) and plays a vital role in cold tolerance by eliminating methionine sulfoxide and reactive oxygen species, which accumulate in the endoplasmic reticulum during cold acclimation (22). Recent study found that MSRB3 deficiency induces apoptosis in breast cancer cells, lung cancer cells and colon cancer cells through p53-independent and ER stress-dependent pathways $(23,24)$. CENPM, also known as proliferation-associated nuclear element 1 , is a protein encoded by the CENPM gene in humans, and is involved in centromere assembly and immune responses (25). Human CENPM is mainly present in immune cells of tumor tissues of leukemias and lymphomas and in tumor-derived cell lines (26). In addition, CENPM participates in the formation of complexes in the nucleoplasm of viable human breast cancer cells outside centromeres (27). ZIC2 is located on chromosome 13q32, and its amino acid sequence is highly conserved; a previous study has found that loss of heterozygosity in the 13q32 region of ZIC2 leads to anterior cerebral schizophrenia (28). It has been reported that ZIC2 is a critical regulator of Kaposi's sarcoma-associated herpesvirus latency maintenance (29). Marchini et al (30) found that ZIC2 overexpression was closely related to proliferation and metastasis of ovarian cancer cells. Additionally, ZIC2 plays an indispensable role in cell proliferation and apoptosis in pancreatic ductal adenocarcinoma (PDAC) (31) and hepatocellular carcinoma, and ZIC2 is a potential therapeutic target in PDAC (32). In summary, these three genes play almost completely different biological roles in different types of cancers, however these have not been validated in further functional and mechanistic experiments in cervical cancer.

In the present study, a three-gene signature was established and identified as an independent prognostic factor for 
patients with cervical cancer. The Cox coefficient based on univariate Cox regression was obtained and established a risk score. In addition, ROC analysis was used to determine the optimal cutoff for classifying high- and low-risk patients. In the univariate Cox regression model, patients with high-risk scores had significantly shorter survival times. The expression of the MSRB3, CENPM and ZIC2 genes was significantly associated with the OS rate of patients with cervical cancer $(\mathrm{P}<0.05)$, revealing that these three genes include both protective factors and risk factors. These results indicate that genes play a key role in the progression and prognosis of cervical cancer. The present study has the advantage of using large databases, complete clinical data, and good sample quality control procedures and provides novel ideas for tumor-targeted therapy. The limitations of the present study are that the gene expression level data obtained from TCGA database may not fully represent the expression of MSRB3, CENPM and ZIC2 at the protein level. Subsequent studies require further experiments to validate these results and further analysis, including immunohistochemistry assays. To make the present study more clinically meaningful, it is necessary to study the three target genes and their related signaling pathways via functional experiments; in clinical terms, further association between these genes and the prognosis of patients with cervical cancer needs to be evaluated from clinical data. Therefore, cervical tumor specimens and clinical prognosis information will be collected from The Affiliated Hospital of Zunyi Medical University) and verify the results through specific experiments in our hospital oncology laboratory. In addition, the development of biomarkers usually includes the establishment of models such as training sets, validation sets and test sets. This model was not built in the present study as the literature states it is not suitable for small sample data. Since the sample is relatively small, it is not suitable to randomly divide data into three parts for verification when setting up grouping. Finally, statistical analysis showed that the P-value of the survival curves of each group was not statistically significant, and the AUC value of the ROC curve was relatively small. When the sample is small, the criteria for establishing the three models are not enough and the results are less accurate. In conclusion, the present study used TCGA database and found that MSRB3, CENPM and ZIC2 can be used as prognostic biomarkers for cervical cancer, thus providing novel ideas and targets for future research on cervical cancer. This research belongs to the research field of biological information prediction. The main purpose is to provide prima facie evidence for the future assessment of patient risk and prognosis, but further verification is required from further investigation and clinical experiments to improve its accuracy.

In summary, our study shows that the identified three-gene signature can be used not only as a prognostic indicator for cervical cancer but also as a predictor of patient risk. In addition, further validation by functional experiments combined with clinical trials are required, for application of the threegene signature in clinical practice for the benefit of patients with cervical cancer.

\section{Acknowledgements}

Not applicable.

\section{Funding}

This study was financially supported by the Natural Science Foundation of China (grant nos. 81360351) and grants from the National Natural Science Foundation of China (grant no. 81560407).

\section{Availability of data and materials}

Data sets used and/or analyzed during the current study are available in the TCGA database (https://www.cancer.gov/aboutnci/organization/ccg/research/structural-genomics/tcga).

\section{Authors' contributions}

TTD, HM and JHF conceived and designed the study. TTD and HM conducted the database mining. HM and JHF analyzed data and compiled charts. TTD wrote the manuscript and approved the final version.

\section{Ethics approval and consent to participate}

Not applicable.

\section{Patient consent for publication}

Not applicable.

\section{Competing interests}

The authors declare that they have no competing interests.

\section{References}

1. Liang B, Li Y and Wang T: A three miRNAs signature predicts survival in cervical cancer using bioinformatics analysis. Sci Rep 7: 5624, 2017.

2. Siegel RL, Miller KD and Jemal A: Cancer statistics, 2018. CA Cancer J Clin 68: 7-30, 2018.

3. Bray F, Ferlay J, Soerjomataram I, Siegel RL, Torre LA and Jemal A: Global cancer statistics 2018: GLOBOCAN estimates of incidence and mortality worldwide for 36 cancers in 185 countries. CA Cancer J Clin 68: 394-424, 2018.

4. Pimple S, Mishra G and Shastri S: Global strategies for cervical cancer prevention. Curr Opin Obstet Gynecol 28: 4-10, 2016.

5. Li H, Wu X and Cheng X: Advances in diagnosis and treatment of metastatic cervical cancer. J Gynecol Oncol 27: e43, 2016.

6. Bahrami A, Hasanzadeh M, Shahidsales S, Farazestanian M, Hassanian SM, Moetamani Ahmadi M, Maftouh M, Gharib M, Yousefi Z, Kadkhodayan S, et al: Genetic susceptibility in cervical cancer: From bench to bedside. J Cell Physiol 233: 1929-1939, 2018.

7. Guido R: Cervical cancer screening. Clin Obstet Gynecol 61: 40-51, 2018.

8. Clough E and Barrett T: The gene expression omnibus database. Methods Mol Biol 1418: 93-110, 2016.

9. Chandran UR, Medvedeva OP, Barmada MM, Blood PD, Chakka A, Luthra S, Ferreira A, Wong KF, Lee AV, Zhang Z, et al: TCGA expedition: A data acquisition and management system for TCGA data. PLoS One 11: e0165395, 2016.

10. Park S, Eom K, Kim J, Bang H, Wang HY, Ahn S, Kim G, Jang H, Kim S, Lee D, et al: MiR-9, miR-21, and miR-155 as potential biomarkers for HPV positive and negative cervical cancer. BMC Cancer 17: 658, 2017.

11. Oliveira CA, Russomano FB, Gomes Júnior SC and Corrêa Fde M: Risk of persistent high-grade squamous intraepithelial lesion after electrosurgical excisional treatment with positive margins: A meta-analysis. Sao Paulo Med J 130: 119-125, 2012. 
12. Li Q, Su YL and Shen WX: A novel prognostic signature of seven genes for the prediction in patients with thymoma. J Cancer Res Clin Oncol 145: 109-116, 2019.

13. Hou C, Zhuang Z, Deng X, Xu Y,Zhang P and Zhu L: Knockdown of Trio by CRISPR/Cas9 suppresses migration and invasion of cervical cancer cells. Oncol Rep 39: 795-801, 2018.

14. Li W, Liang J, Zhang Z, Lou H, Zhao L, Xu Y and Ou R: MicroRNA-329-3p targets MAPK1 to suppress cell proliferation, migration and invasion in cervical cancer. Oncol Rep 37: 2743-2750, 2017.

15. Ferlay J, Soerjomataram I, Dikshit R, Eser S, Mathers C, Rebelo M, Parkin DM, Forman D and Bray F: Cancer incidence and mortality worldwide: Sources, methods and major patterns in GLOBOCAN 2012. Int J Cancer 136: E359-E386, 2015.

16. Di J, Rutherford S and Chu C: Review of the cervical cancer burden and population-based cervical cancer screening in China. Asian Pac J Cancer Prev 16: 7401-7407, 2015.

17. Farzaneh E, Heydari H, Shekarchi AA and Kamran A: Breast and cervical cancer-screening uptake among females in Ardabil, northwest Iran: A community-based study. Onco Targets Ther 10 : 985-992, 2017.

18. Moyer VA and U.S. Preventive Services Task Force: Screening for cervical cancer: U.S. Preventive Services Task Force recommendation statement. Ann Intern Med 156: 880-891, 2012.

19. Huh WK, Ault KA, Chelmow D, Davey DD, Goulart RA, Garcia FA, Kinney WK, Massad LS, Mayeaux EJ, Saslow D, et al: Use of primary high-risk human papillomavirus testing for cervical cancer screening: Interim clinical guidance. Obstet Gynecol 125: 330-337, 2015.

20. Nakada T, Akiba T, Yabe M, Tanaka K, Nakano M, Suzuki M and Morikawa T: Clinicopathological features of thymoma with ring calcification: Case reports. Ann Thorac Cardiovasc Surg 23: 256-261, 2017.

21. Kim HY: The methionine sulfoxide reduction system: Selenium utilization and methionine sulfoxide reductase enzymes and their functions. Antioxid Redox Signal 19: 958-969, 2013

22. Lee E, Kwak GH, Kamble K and Kim H: Methionine sulfoxide reductase B3 deficiency inhibits cell growth through the activation of p53-p21 and p27 pathways. Arch Biochem Biophys 547: $1-5,2014$.

23. Kwak GH and Kim HY: MsrB3 deficiency induces cancer cell apoptosis through p53-independent and ER stress-dependent pathways. Arch Biochem Biophys 621: 1-5, 2017.

24. Kwak GH, Kim TH and Kim HY: Down-regulation of MsrB3 induces cancer cell apoptosis through reactive oxygen species production and intrinsic mitochondrial pathway activation. Biochem Biophys Res Commun 483: 468-474, 2017.
25. Liu C, Zhang W, Yang D and Liu Y: Molecular characterization, polymorphism, and association of porcine GADD45G Gene. Anim Biotechnol 26: 230-236, 2015.

26. Oliveras-Ferraros C, Vazquez-Martin A, Cuyàs E, CorominasFaja B, Rodríguez-Gallego E, Fernández-Arroyo S, Martin-Castillo B, Joven J and Menendez JA: Acquired resistance to metformin in breast cancer cells triggers transcriptome reprogramming toward a degradome-related metastatic stemlike profile. Cell Cycle 13: 1132-1144, 2014.

27. Hoischen C, Yavas S, Wohland T and Diekmann S: CENP-C/ $\mathrm{H} / \mathrm{I} / \mathrm{K} / \mathrm{M} / \mathrm{T} / \mathrm{W} / \mathrm{N} / \mathrm{L}$ and hMis12 but not CENP-S/X participate in complex formation in the nucleoplasm of living human interphase cells outside centromeres. PLoS One 13: e0192572, 2018.

28. Sutherland MJ, Wang S, Quinn ME, Haaning A and Ware SM: Zic3 is required in the migrating primitive streak for node morphogenesis and left-right patterning. Hum Mol Genet 22: 1913-1923, 2013

29. Lyu Y, Nakano K, Davis RR, Tepper CG, Campbell M and Izumiya Y: ZIC2 is essential for maintenance of latency and is a target of an immediate early protein during Kaposi's sarcoma-associated Herpesvirus lytic reactivation. J Virol 91: pii: e00980-17, 2017.

30. Marchini S, Poynor E, Barakat RR, Clivio L, Cinquini M, Fruscio R, Porcu L, Bussani C, D'Incalci M, Erba E, et al: The zinc finger gene ZIC2 has features of an oncogene and its overexpression correlates strongly with the clinical course of epithelial ovarian cancer. Clin Cancer Res 18: 4313-4324, 2012.

31. Inaguma S, Ito $\mathrm{H}$, Riku M, Ikeda $\mathrm{H}$ and Kasai K: Addiction of pancreatic cancer cells to zinc-finger transcription factor ZIC2. Oncotarget 6: 28257-28268, 2015.

32. Zhu P, Wang Y, He L, Huang G, Du Y, Zhang G, Yan X, Xia P, Ye B, Wang S, et al: ZIC2-dependent OCT4 activation drives self-renewal of human liver cancer stem cells. J Clin Invest 125: 3795-808, 2015.

This work is licensed under a Creative Commons Attribution-NonCommercial-NoDerivatives 4.0 International (CC BY-NC-ND 4.0) License. 